\title{
Ueber Lichen ruber acuminatus, planus und Pityriasis pilaire.
}

\author{
Von \\ Prof. Neumann in Wien.
}

(Hiezu Tafel I-III.)

Die Geschichte des Lichen ruber hat in den letzten zwei Decennien vielfache Wandlungen durchgemacht und steht noch derzeit auf dem Programme der Discussion, ohne dass es bisher gelungen wäre, dieselbe einer definitiven Entscheidung näher zu rücken.

Während noch bis zum Jahre 1873 nur der von Hebra beschriebene Lichen ruber gekannt und der Lichen planus von Erasmus Wilson, T. Fox wenigstens hierzulande nur aus der Literatur geläufig war, während $\mathrm{E}$. Wilson selbst den Lichen ruber (Hebra) nicht kannte, und Hebra anfangs den Lichen ruber für eine absolut tödtliche Affection erklärt hatte und erst später gleich den übrigen Dermatologen zu einer anderen Ansicht gelangt war, indem er den Arsenik geradezu als ein Specificum gegen diese Krankheit adoptirt hatte, leuguen Andere wie Piffard, Brocque, Rona die Existenz des Lichen acuminatus vollständig, während Taylor (The New-York med. Journal 1889) behauptet, der Lichen planus gehöre gar nicht zum Lichen ruber, sei eine Erkrankung sui generis.

Durch die französische Schule endlich wurde die Pityriasis pilaire beschrieben, von welcher Einzelne behaupten, sie sei nichts Anderes als der Lichen ruber acuminatus. 
Da nunmehr die ganze Lichenfrage derzeit in Verwirrung gebracht und namentlich mit der Frage der Existenz der Pityriasis pilaire verquickt wurde, will ich es versuchen, letztere an der Hand der klinischen Beobachtung und der mikroskopischen Untersuchung näher zu erörtern.

Die ganze Frage gipfelt in Folgendem: 1. Gibt es einen Lichen ruber im Sinne Hebra's (dem Kaposi das Epitheton acuminatus gegeben)? 2. Gibt es einen Lichen planus (E. Wilson)? 3. Ist die Existenz der Pityriasis pilaire erwiesen? Bezüglich der ersten Frage, der Existenz des Lichen ruber scheint es überflüssig, weitere Beweise zu erbringen. Die Symptome der Erkrankung sind von Hebra so classisch beschrieben worden, die anatomischen Untersuchungen (Neumann, Sitzungsbericht der k. Akademie d. Wissensch. 1869, die Untersuchungen Biesiadecki an d. patholog.-anatom. Institute) haben für diese Erkrankung Merkmale ergeben, welche die Existenz des Lichen ruber über jeden Zweifel erheben.

Anders verhält es sich mit dem Lichen planus. Dieser war uns wenigstens in der Wiener Schule bis zum Jahre 1870 vollständig unbekannt geblieben. Da die Efflorescenzen sich häufig am männlichen Genitale zuerst localisiren, wurde die Erkrankung auch wegen ihrer Configuration für Lichen syphiliticus gehalten. Die mattweiss gefärbtenKnötchen, welche in periphererAnordnung hier das auffallendste Symptom bilden, lassen es plausibel erscheinen, dass ich die Krankheit zu jener Zeit anfangs unter dem Namen Herpes chronicus (S. Lehrbuch, 3. Auflage) später (Vierteljahresschrift für Dermatologie und Syphilis 1875) als Dermatitis herpetiformis beschrieben habe.

Bei beiden Bezeichnungen hatte ich stets die peripheren mattweissen Efflorescenzen vor Augen. Als später 1877 mein Collega Kaposi einen Krankheitsfall in der Gesellschaft der Aerzte demonstrirte, konnte ich eine Illustration, die C. Heitzmann im Jahre $1873 \mathrm{mir}$ angefertigt, vorzeigen und war ebensowenig wie heute im Zweifel, dass das von mir beschriebene Krankheitsbild genau mit dem von E. Wilson unter dem Namen Lichen planus beschriebenen ïbereinstimme. Allerdings fehlt bei Letzterem noch die genaue anatomische Untersuchung. Ich verweise hier auf meine Darstellung (Siehe 
diesen Artikel 1875), aus Felcher ich Folgendes entnehme. Es heisst daselbst: Die Krankheit entwickelt sich unter Bildung von hanfkorngrossen blaugerötheten, im Centrum bläulichweiss gefärbten Efflorescenzen, von denen aus sich die Röthung und Infiltration gegen die Peripherie allmälig weiter verbreitet. Die Efflorescenzen werden selbst thalergross und sind an denselben die punktförmigen Entfärbungen besonders deutlich entwickelt, während die Mitte mehr gleichmässig roth erscheint; sie stehen theils einzeln, theils confluiren sie, rücken oft mit ihrer Wandung an einander und erscheinen theils scheibenförmig, theils gyrirt, wobei das Centrum entweder noch weisse Punkte zeigt oder mehr gleichförmig geröthet und mit Schuppen bedeckt erscheint. Die grösste Aehnlichkeit haben diese weissen Punkte mit den Eczembläschen an der Planta pedis und Palma manus, bei welchen das Exsudat die Epidermis noch nicht emporgehoben oder durchbrochen hat. Mit der Dauer der Erkrankung nimmt auch die Schuppenmenge beträchtlich zu; an einzelnen Hautstellen finden sich harte, dicht an und übereinander gelagerte, braun gefärbte, fest anliegende Lagen von Schuppen und Schildern, die mit ihrer Unterlage in festem und innigem Contacte sind und nach deren Entfernung eine excoriirte verdickte Hautpartie zum Vorschein kommt; an anderen Körperstellen (Stamm) erreicht die Schuppenmenge gleichwie das Infiltrat nie diesen Umfang. Mit dem mehrmonatlichen Bestande des Leidens nimmt das Infiltrat ab und es bleibt, wenn dasselbe geschwunden ist, ein dunkelbraun pigmentirter Fleck oder gleich pigmentirte eingesunkene Punkte zurück. Sich selbst überlassen, kann die Krankheit jahrelang bestehen und sich mit neuen Eruptionen weiter verbreiten, wobei die älteren sich spontan zurückbilden und zwar in der Art, dass die Verdickung und Infiltration allmälig abnehmen, die Schuppen sich loslösen und eine weiche, nichtnarbige pigmentirte Partie hinterlassen, wobei auch das Jucken vollständig schwindet. Ist hier nicht ganz genau das klinische Bild des Lichen planus gegeben?

Wegen dieser punktförmigen bläschenartigen Efflorescenzen hat Unna viel später diese Erkrankung als L. miliaris bezeichnet. 
Anatomisch wäre besonders Folgendes hervorzuheben: Beim Lichen planus sind es mehr die Schweissdrüsen, welche pathologisch verändert sind, beim Lichen ruber (acuminatus) (Siehe Sitzungsbericht der k. Akad. d. Wissenschaften) mehr die Haarbälge, welche einen mehr acinösen Bau annehmen, konische Zapfen darbieten, in denen die Zellen der äusseren Wurzelscheiden vermehrt sind.

Die Vergrösserung der Papillen und die Zellwucherung, zumal in den Gefässen der oberen Cutislagen sind bei Lichen acuminatus deutlich ausgeprägt.

Beim Lichen planus findet man eine excessive Bildung der Epidermis. Die Zunahme betrifft die verhornten Zellen besonders und das ganıe Rete Malpighii, dessen Lagen beträchtlich zugenommen haben. Die Zellen des Rete Malpighii sind besonders um den Kern körnig getrübt und geschwellt; sie sind zumeist Stachelzellen.

Die Papillen sind im Breiten-, noch mehr im Höhendurchmesser vergrössert (siehe l. c. die Tafeln), ihre Gestalt theils konisch, theils zugespitzt und vielfach verzweigt; ihr Gewebe mit netzförmig angeordneten körnigen Wucherungen versehen. Die Gefässschlingen sind breiter, deren Adventitia mit Wucherungen versehen, in der Papille selbst vielfach gewunden; gedrängter finden sich die Wucherungen unter den Papillen an den beträchtlich erweiterten Gefässen. Im Cutisgewebe sind die Wucherungen u. zw. nur im oberen Theile in grösseren Inseln und Zügen, gleichwie in Form von Netzen angeordnet. Die Haar- und Talgfollikel sind normal, die glatten Muskelfasern hypertrophisch. Die Wandungen der Schweissdrüsen, der Austührungs- und Drüsengänge sind durch Zunahme der Inhaltszellen verbreitert, durch Schwellung der Wandungen und selbst durch körnige Wucherungen, welche die Schweissdrüsenknäuel umgeben und auch den Drüsengang begleiten. Die Enchymzellen im Ausführungsgange sind vermehrt; in den Drüsenknäueln findet man zahlreiche runde opake, das Licht stark brechende Colloidezellen, während in den bereits vorgeschrittenen Stadien der Krankheit die Schweissdrüsengänge mit verhornten Zellen gefuillt und in den tiefsten Stellen 
dunkelbraun pigmentirte Zellen enthalten. In den späteren Stadien der Krankheit finden sich pigmentirte Zellen, körniges, rostbraunes bis schwarzes Pigment im oberen Theile des Cutisgewebes, in den Papillen, in der Adrentitia der Gefässe in reicher Menge enthaltend. Die neueren Arbeiten von Weyl (Ziemssen, Handbuch der Hautkrankheiten 1883), Köbner (Berliner klinische Wochenschrift 1887), Bender (Deutsche medicinische Wochenschrift 1887), Robinson (Journal of cut. and genit. urin. diseas. 1889), Philippson (Monatshefte für praktische Dermatologie 1889), Crocker (Diseas. of the Skin 1888), Török (Monatshefte für Dermatologie, B. VIII) haben im Wesentlichen keine Befunde ergeben, welche wesentlich hier die meinen alterirt hätten. Nach Kaposi dagegen stimmen die histologischen Veränderungen, der Verlauf und das therapeutische Verhalten beider Lichenformen mit einander überein, während Robinson und Taylor selbe gerade aus anatomischen Gründen auseinander halten.

Wir kommen nun zum Kernpunkte der ganzen Frage, zum Verhältniss des Lichen ruber zur Pityriasis pilaire.

Bisher mussten wir die Führung in dieser Frage der französischen Schule überlassen. Diese wurde durch amerikanische und englische Aerzte unterstiutzt; in Norwegen war es C. Boeck, in Wien H. v. Hebra, Unna in Hamburg welche für die Existenz dieser Krankheit eingetreten sind.

Beim jüngsten Congresse in Paris stand die Pityriasis pilair an der Spitze der Discussion (Siehe Congrès intern. de Dermat. et de Syph. 1889, Comptes rendus, Paris 1890, pag. 16) und es betheiligten sich an dieser Kaposi, Jamieson, M. Schwimmer, M. Hallopeau, จ. Hebra, C. Boeck, M. Unna, M. Malcolm Morris, Dubois Havenith, Leloir, Schiff, Besnier, De Amicis, Vidal; und ausser Kaposi war Niemand gegen die Pityriasis pilaris als selbständige Erkrankung aufgetreter.

Vielleicht wird nachfolgender Fall, der seit 18 Monaten in meiner Klinik in Behandlung steht, einiges zur Klärung der Pityriasis-Frage beitragen.

Näheres über Pityriasis pilaris lesen wir bei E. Besnier (Annales de Dermatologie et Syphilid. 1869), welcher diese Erkran- 
kung zuerst wissenschaftlich bearbeitet; weiters und unter seiner Leitung Richaut 1877 und Brocque (Arch. génerale de Médic. 1884); aber schon bei Rayer 1835 begegnet man derselben Erkrankung unter der Bezeichnung Psoriasis général apparence particulière de la desquamation sur les points occupés par les poils), ebenso bei Devergie (Traité pratique de maladies de la peau, Paris 1856). Ausserdem sind es die Arbeiten von Robinson, Piffard, Taylor, die Beobachtung von Malcolm Morris und die literarischen Zusammenstellungen, wie selbe H. v. Hebra, Unna, Török, Rona gemacht, die zur Klarstellung der Frage beigetragen haben. Es wird von allen Beobachtern als das prägnanteste Merkmal hervorgehoben: Schuppen, deren Sitz an den Mündungen der Follikel (Asperité des orific. folliculaire), gleichwie die Reihenfolge der Knötchenbildung.

Ich will nunmehr den Fall meiner Klinik vorführen:

H. S., 33 Jahre alt, wurde am 19. März in meine Klinik aufgenommen. Der Kranke klein, mässig genährt, vor 8 Jahren war er an Cystitis erkrankt.

Die dermalige Affection besteht seit 14 Tagen:

An den unteren Extremitäten finden sich theils linsekorngrosse, theils punktförmige geröthete, über das Niveau der Haut mässig erhabene, den Haarbälgen entsprechende, an ihrer Oberfläche glatte Efflorescenzen.

Stellenweise sind mehrere derartige Knötchen auseinander getreten in der Ausdehnung von Linsengrösse; es lässt sich die Epidermis von denselben als eine dünne Lamelle ablösen und erscheint darunter die Haut abgeblasst, weich, nicht infiltrirt.

An den Schulterblättern, am Nacken und theilweise an der Brustwand sind namentlich durch Confluenz blassrothe, mässig elevirte, nicht infiltrirte grössere Flächen zu sehen.

An dem Handrücken sind die Furchen und Linien tief, stehen weit von einander ab und sind denselben entsprechend zahlreiche seichte Einrisse. An den ersten Phalangen entsprechend den Haaren findet man mehr als hirsekorngrosse, mässig derbe, im Centrum sich bereits abschuppende Knötchen.

Die Nägel mit mässiger Bildung von hypertrophischer Nagelsubstanz.

Die Innentläche der Vorderarme und die Ellenbeuge zeigen dunkelrothe Verfärbung der Haut, stellenweise zahlreiche Petechien.

Die Gesichtshaut gespannt, trocken; die Augenliderhaut verdickt, die obere Furche besonders vertieft, beim Schliessen der Lider wird der Bulbus nicht vollständig bedeckt. 
Die Fusssohlen beiderseits geschwellt, gegen den Fussrücken dunkelroth mit zahlreichen lämorrhagischen Punkten versehen.

21. März: Der Kranke klagt über Frösteln, Nacht schlaflos.

25. März: Die Erscheinungen haben sich nicht wesentlich geändert.

1. April : Die Haut der Hände mehr geschwellt, neben den horizontalen awch längliche Fissuren, die den Metakarpalzwischenräumen entsprechen.

Die zahlreichen Knötchen an den unteren Extremitäten und am Stamme sind lividroth, theilweise hämorrhagisch, theils confluirt; ersteres an den Unterschenkeln, letzteres am Stamme.

Die Schwellung der Haut geringer und mehr eine diffuse, flächenförmige Ausbreitung zumal im Gesichte, Brtistwand, Nacken und Rücken.

Die Haut selbst weich, elastisch, nur an jenen Partien, an denen die Effiorescenzen erst später aufgetreten sind, z. B. an den Streckflächen des Ellbogengelenkes ist dieselbe noch verdickt infiltrirt, mehr dunkelroth.

Die Röthung hat sich auf den ganzen Fussrücken bis über die Malleoien ausgebreitet und erscheint daselbst mehr hell, die breiteren Flecke mehr blauroth.

9. April: Brust, Bauchwand und Rückenhaut abgeblasst, in geringerem Grade ödematös. Vorne die Abschuppung bereits abgeiaufen, rückwärts haften noch theils kleienförmige, theils linsengrosse hellweisse Schuppen an.

An den Extremitäten u. z. am Handrücken sind die Schuppen abgestossen, an den Phalangen bilden sich noch lederartige, fest anhaftende ringförmige zerklüftete Schuppen, an den Handtellern sind dieselben noch diffus aufgelagert, fest anhaftend; jene Stellen an der Beugefläche des Ellbogengelenkes, die noch vor Kurzem dunkelroth hämorrhagiseh gefärbt waren, sind nunmehr blassroth, die Haut weich und elastisch ohne jede ödematöse Schwellung, ebensowenig ist an irgend einer Stelle noch ein Knötchen zu sehen.

An der unteren Extremität stellenweise noch abschuppende, an den Fusssoblen noch anhaftende Schollen von Epidermis (Hyperkeratose). Fussrücken und Oberschenkel leicht geröthet, an den Untersehenkeln, woselbst die Abschuppung noch nicht begonnen erscheint, die Haut etwas braunroth gefärbt, und beim Fingerdrucke fndet sich unter der gedrückten braunrothen Stelle eine grünlichgelbe Verfärbung von ausgetretenem Biutfarbstoffe.

Auch einzelnstehende abgeflachte lividrothe Knötchen sind hier zu sehen. Stirnbaut rauh, uneben, desgleichen der grösste Theil der Wange und der Ohrmuscheln. Ueber Kinn und Augenbrauenbogen, und dem os-zygomaticum sind perlmutterartig glänzende, fest anhaftende Schuppen aufgelagert.

22. April: Die Infiltration an der ganzen Hautoberfläche geschwunden, die Röthung geringer, die Schuppen sind dünner und in grösseren Mengen aufgelagert. Auf das Gesicht und die Kopfhaut bekommt Patient Vaselin, auf die Füsse und Hände Unguent. diachyl. alb. 
30. April: Die Infiltration total geschwunden.

1. Mai: Bis auf wenige Stellen an den Extremitäten, die mit Schuppen bedeckt sind, ist die Haut, namentlich im Gesichte, ganz normal. Patient wurde aus meiner Klinik entlassen.

Der ganze Process dauert bis heute 48 Tage und kann bis auf geringe Reste der Entzündung nahezu als abgelaufen erklärt werden. Während dieser Zeit hat der Kranke an Körpergewicht zugenommen, das Allgemeinbefinden war nicht gestört. Ich habe am 4. April 1890 (siehe Sitzung der dermatol. Gesellschaft) den Kranken mit der Diaguose Erythema toxicum vorgestellt, und die Discussion, die sich an diese Demonstration geknüpft, an der sich unter Anderen Kaposi und H. v. Hebra betheiligten, war einmäthig in der Annahme dieser Diagnose. In dem Berichte heisst es: Herr Kaposi hat diesen sehr interessanten Fall gesehen und sofort Erythema toxicum diagnosticirt. Wir werden im Verlaufe der später folgenden Auseinandersetzungen sehen, dass wir mit gleich grosser Entschiedenheit diese Diagnose wieder fallen gelassen und dass die Krankheit von uns (Hebra ausgenommen) als Lichen ruber acuminatus erklärt haben. Denn schon 24 Tage nach seiner Entlassung kam Patient wieder wegen Urethritis mit folgenden Erscheinungen: Der Stamm blassroth mit zahlreichen disseminirten punktförmigen, an der Oberfläche mit fest anhaftenden hellweissen Schüppchen versehenen Knötchen, welche sich bei Berührung reibeisenartig anfühlten und durch den kratzenden Nagel oder durch Reiben mit Alkohol entfernt werden können. In der Crena ani und ad nates, namentlich an ersterer, die Schuppenmenge eine beträchtlichere, die Schuppen perlmutterartig glänzend, die Haut mässig infiltrirt.

Solche Knötchen von etwas grösserem Umfange finden sich auch am Handrücken und an den Vorderarmen, an ersteren, namentlich über der Phalange sind dieselben dicht gedrängt, in Gruppen angeordnet, mit perlmutterartig glänzenden Schuppen bedeckt.

An den Fusssohlen ist die Epidermis schwielig verdickt. Die Nägel zumal gegen das freie Ende hin mattweiss, gegen die Wurzeln noch geröthet.

Während die recenten Knötchen von einem rothen Hofe begrenzt, schon nach wenigen Tagen mit confluirenden Schüppchen bedeckt erschienen, sind andere äItere persistent, derb, an der Oberfläche rauh, reibeisenartig.

Die hier angeführten Symptome haben demnach vorwiegend für Lichen ruber acuminatus gesprochen und ich bin für diese Diagnose in der dermatolog. Gesellschaft mit Wärme eingetreten.

Aber es sollte anders werden.

Die Schwankungen, denen wir Alle, die sich für diesen Krankheitsfall interessirten, ausgesetzt waren, sind wenigstens plötzlich gewichen, denn schon am 28. Juni hatte die Zahl der Knötchen zugenommen. 
An der Streckfläche der Ellbogengelenke, am Nacken, ad nates, den äusseren Flächen der Ober- und Unterschenkel, über der Fossa poplitea sind die Knötchen dicht gedrängt, mit Schuppen bedeckt. Die Auflagerungen unter der Nagelsubstanz haben zugenommen. Die Beweglichleit der Finger beträchtlich eingeschränkt, so dass diese in halbgebeugter Stellung sind.

Am 29. Juni wird Borsalbe am rechten Vorderarme applicirt and schon am 9. Juli waren die Knötchen über den Phalangen, welche stecknadelkopfgross waren, abgeflacht.

Am linken Vorderarme, woselbst die Haut unbehandelt geblieben, war dieselbe Rauhigkeit und Derbheit wie zuvor.

Die Rückenhaut abgeblasst, schuppig. Ad nates, in der Crena ani perlmutterartig glänzende dicke Epidermisauflagerungen.

14. Juli: Gesichtshaut und Ohrmuscheln mit fest anhaftenden perlmutterartig glänzenden Schuppen bedeckt.

Gleiche Veränderung bieten Nacken, nates, Crena ani dar.

An den Lenden sind die Furchen und Linien tief, weiter von einander abstehend, eine genaue Begrenzung der Peripherie, wie solche ausnahmslos beim Lichen ruber vorkommt, ist hier jedoch nicht zu bemerken. Die Hyperkeratose an der Vola manus und Planta pedis besteht noch fort. Die hirsekorngrossen Knötchen an den Fingern sind abgeflacht und an ihrer Stelle grössere Schuppenlamellen aufgelagert.

18. Juli. Am Handrücken beiderseits die Haut schuppig, rechts, woselbst die Borsalbe applicirt war, abgeflacht, links am freien Handrücken noch einzelne hirsekorngrosse, abgeflachte Knötchen. Ueber den ersten Phalangen sind die Knötchen geschwunden, nur angedeutet durch einen weissen Epidermissaum.

21. Juli: Der Kranke, welcher bisher 200 Pil. asiatic. genommen, klagt über Athemnoth, in der Herzgegend ein stechender Schmerz; Intoxicationserscheinungen infolge des Arsenikgebrauches.

26. Juli: Die Beschwerden haben nachgelassen. Es zeigt nunmehr die Gesichtshaut zumal die behaarten Stellen der Oberlippe, die behaarten Stellen der Wange und des Kinnes, der Ohrmuscheln mit diffusen, perlmutterartig glänzenden Schuppen bedeckt. An jenen Stellen, woselbst die Schuppen abgefallen sind, die Schuppenlamellen verdünnt, in dem. Centrum anhaftend, in der Peripherie leicht abgelöst und am Stamme sind an jenen Stellen, welche noch vor 8 Tagen geröthet waren punktformige, über das Niveau der Haut erhabene, der Cutis anserina entsprechende Efflorescenzen, welche im Centrum mit dünnen, punktförmigen Schüppchen bedeckt sind.

An der Rückenhaut sind die Furchen und Linien mässig vertieft. Die Epidermis löst sich in kleienförmigen Massen ab. Ad nates bilden die Schuppen grosse Rasen von perlmutterartigem Glanze, wobei jedoch die Haut selbst nicht infiltrirt erscheint. Die Finger in halbgebeugter Stellung, vollständig active Streckung unmöglich, ehenso unmöglich ist die passive Bewegung. An den ersten Phalangen, wos elbst vorher hirsekorngrosse, 
gruppirte, den Haaren entsprechende Knötchen sassen, finden sich nunmehr nur diffuse flächenförmige Ausbreitungen von Schuppen, unter welchen. die Haut theils blassroth, theils normal gefärbt ist, glatt, nicht verdickt erscheint. An der Hohlhand und Fusssohlen Hyperkeratose mit Rhagaden.

Die Nägel, in ihrer Form nicht verändert, zeigen von dem Nagelbette ausgehende neugebildete Nagelsubstanz, während der Nagel selbst intact ist.

9. August: Die Knötchen an den Ober- und Unterschenkeln vollständig verschwunden. Die Epidermis an den Unterschenkeln in grösseren Lamellen abschuppend, trocken; kleinere Knötchen finden sich an den Oberschenkeln.

An der rechten Sohle eine leicht blutende Rhagade, links in der inneren Malleolengegend eine etwas kleinere.

11. August: Haut an den unteren Extremitäten geröthet, Schuppen daselbst verschwunden.

14. August: Knötchen an der Brust, besonders die einzeln stehenden verkleinert.

16. August: Patient 46 Kilogramm schwer. Im Ganzen hat die Ernährung und sein Körpergewicht nicht abgenommen. An der Haut des Rückens und der Bauchwand ist die Haut blass geröthet, an dem ersteren schon mehr mit schmutzig weissen, derberen, mehr den Knoten ähnlichen Auflagerungen bedeckt. An der unteren Hälfte der Brustwand finden sich einzelne normal weiss gefärbte Stellen und in diese eingehend zahlreiche punktförmige, schmutzigbraun gefärbte, disseminirte Knötchen, welche an der Oberfläche zum grössten Theile glatt, stellenweise jedoch mit dünnen Schuppchen bedeckt sind. Die Haut erscheint überall weich, elastisch, nicht infiltrirt. Intensivere Veränderungen zeigen noch die Zehen und Nagelbett, an welchen grössere Schuppenlamellen vorkommen.

Gesichtshant, Ohr und Nacken noch schuppig.

5. September: Der Kranke wägt $48 \mathrm{~kg}$. Die Haut an den Extremitäten mit dünnen, beim Reiben theils kleienförmigen, leicht anhaftenden, theils linsengrossen, leicht abfallenden Schuppen bedeckt, unter denen die Haut theils bereits normal weich, dünn und elastisch, theils geröthet erscheint.

An den Vorderarmen, namentlich woselbst jie Zahl der ranhen reibeisenartig sich anfühlenden Knötchen eine beträchtlichere war, sind dieselben vollständig geschwunden, ebenso über den ersten Phalangen vollständig glatt erscheint.

Am Stamme ist die Haut geröthet; es finden sich punktförmige, blasse, an ihrer Oberfläche mit dünnen, punktförmigen Epidermismassen bedeckte Knötchen. Die Nägel sind glattrandig, an ibrer Oberfläche zeigen sich längliche, parallel laufende Leisten; der Nagel selbst nicht verdünnt, jedoch von unten her durch neugebildete Massen rom Nagelbette etwas abgehoben.

Die Lymphdrüsen sowohl die Crural- als auch Inguinaldrüsen beider- 
seits vergrössert, die Axillardrüsen rechterseits vergrössert und entzündet, links normal.

Ad nates ist die Haut nicht verdickt, jedoch mit perlmutterartig glänzenden breiten Schuppen bedeckt.

10. September: Seit 6 Tagen wurde Patient mit 0l. jecor. aselli eingepinselt. Die Schuppen sind abgestossen, die Haut vollständig glatt und rechts an der unteren Hälfte der Brustwand sieht man eine flachhandgrosse normal gefärbte Stelle und an dieser sind punktförmige, leicht erhabene Knötchen eingestreut.

Beweglichkeit der Finger wieder vorhanden, Nägel an ihrer Oberfläche glatt bis auf einzelne etwas derbere Leisten und nur im Nagelbette, was namentlich unter dem freien Rande besonders auffällig ist, dichte Massen von Nagelsubstanz.

Die Drüse in der Achselhöhle rechts spontan eröffnet, bereits verkleinert, die anderen bereits beschriebenen Lymphdrüsen haben die oben erwähnte Grösse. Patient bekommt Amylum.

13. October: Die ganze Hautoberfläche geröthet, theils hellroth, theils blassroth, weich elastisch, an der Oberfläche mit Auflagerungen von dünnen weissen, fest anhaftenden Schuppen bedeckt. Gesichtshaut, Hohlhand and Fusssohle gespannt, mit dichteren Lagen verhornter Zellen bedeckt. Pat. bekommt jetzt Theerbäder (2 Stunden), für das Gesicht and Hände Ungu. diachyl. alb.

30. October: Die ganze Hautoberfläche blassroth, die Schuppenbildung hat nachgelassen, die Schuppenauflagerungen an der Hohlhand and Fusssohle, die Schwellung der Haut geringer; und doch zeigt die aufgehobene Hautfalte noch immer eine ödematöse Schwellung in der Tiefe. Die Anflagerung der Nagelsubstanz unter dem Nagel hat abgenommen, wodurch der Nagel nicht mehr so abgehoben aus dem Nagelbette erscheint.

3. November: Haut weich und elastisch, die mimischen Bewegungen des Gesichtes und Bewegungen der Finger frei.

5. November: Die Hautoberfläche ist geröthet nur der Rücken ist blass, die Schuppenbildung am Stamme geschwunden, Haut weich und elastisch, Ohrmuscheln und Kopfhant mit Schuppen bedeckt. Das Mienenspiel ist normal, ebenso ist freie Beweglichleit der Finger nnd Zehen vorhanden. Unter dem Nagel befindet sich keine Auflagerung von Nagelsubstanz mehr, so dass der Nagel aus dem Nagelbette nicht mehr abgehoben erscheint.

1. December: Die Haut dunkel geröthet, in ihrer ganzen Ausdehnung zum Theil mit leicht anhaftenden Schuppen, theils auch mit leicht anhaftendem Exsudate bedeckt. Die Haut am Stamme mattweiss, jedoch ist ein leichtes Oedem vorhanden. Die aufgehobene Hautfalte fühlt sich etwas dicker an. Die Hand u. z. am meisten die Hohlhand mit dichten, schwieligen Epidermismassen bedeckt. Die Nägel an ihrer Oberfläche glatt, an ihrem freien Rande etwas scharfkantig und von dem Nagelbette 
aus eine dünne Lage von Nagelsubstanz vorbanden. Schuppenlagen finden sich auch auf der Fusssohle. An den unteren Extremitäten ist die Haut etwas dicker, namentlich in der Kniekehle. Die Furchen und Linien weit von einander abstehend. Die Affection kommt nunmehr auch auf den behaarten Stellen vor, z. B. an den Schamhaaren, an der Kopfhaut auch an den behaarten Stellen der Gesichtshaut.

9. December: Bis heute bekam Pat. 8 Arsenikeinspritzungen von je 0.005 Acid. arsenic., täglich ein Bad.

Haut an der Bauchwand und stellenweise an der Brust weiss gefärbt, weich elastisch, ebenso an der Vorderfläche der Ober- und Unterschenkel und an den Waden, während an anderen Partien, zumal des Rückens, an den Oberarmen und Kniekehlen noch eine leichte Infiltration der Haut vorhanden ist, gleichwie eine leichte Schuppenbildung die Gesichtshaut noch mit dünnen Krusten bedeckt; geröthet.

12. December: Die ganze Hantoberfäche, welche vor einigen Tagen blass war, ist geröthet, an ihrer Oberfläche theils mit Schuppen, theils mit vertrockneten Exsudatmassen bedeckt, noch etwas ödematös; die Hände beweglicher, dünner; die Nägel bereits im unteren Drittel rosenroth gefärbt, eine äusserst geringe Menge der Nagelsubstanz aus dem Nagelbette hervorwuchernd.

16. Jänner: Nach 34 Arsenikeinspritzungen ist die Haut weich, dünn, blass, Furchen und Linien nicht mehr vertieft, die Epidermisauflagerung geschwunden, die Finger gut beweglich, die Gesichtshant weich, an der Stirne, Nacken- und Gesichtshaut etwas eingesunkene halbkreuzergrosse weisse Stellen, welche atrophisch und pigmentlos sind and einen scharfen Rand haben.

17. Jänner: An der Haut finden sich mehrere überlinsengrosse atrophische, etwas eingesunkene Flecke und in, einzelnen dieser Flecke zeigen sich punktförmige Knötchen, die durch den kratzenden Nagel entfernt werden können. An der linker Brustwand sind 4 solcher Flecke: einer an der Achselfalte und mehrere dicht gedrängt von halb Linsengrösse zwischen den Schulterblättern, 2 am Oberarme rechts und am Fussrücken links. Diese Flecke sind theils in gleicher Flucht mit der umgebenden Haut, theils sind dieselben tiefer eingesunken. An der behaarten Brustwand enthalten dieselben normal gefärbte Haare.

16. Feber: Patient hat bis jetzt 50 Arsenikinjectionen erhalten. An der Haut mehrere $1 / 2$ kreuzer-grosse, unter dem Hautniveau stehende weisse Flecke.

21. Feber: Die Haut allenthalben weich, elastisch, zumeist mit dünnen vertrockneten Exsudatschichten bedeckt.

An der Hohlhand die Epidermisauflagerungen beträchtlich, so dass die Finger in halbgebeugter Stellung erhalten werden und bei passiven Bewegnngen die vollkommene Streckung unmöglich. Am Nagelbette, zumal am freien Rande des Nagels starke Verdickungen der Nagelsubstanz, 
so dass der Rand zwischen dem vorderen Nagelende und der Phalanx mit derselben vollständig ausgefüllt ist.

1. März: Auf der Brust in der Gegend des Nabels an den oberen Extremitäten, an der Stirne am Nacken bis $1 / 2$ lzreuzer-grosse vertiefte, weisse atrophische Stellen.

7. März: Einzelne der eingesunkenen Stellen etwas vergrössert.

15. März: Nach Dampfbädern, Einwickelungen und Vaselin wird die Haut weich und elastisch.

Wir wollen aus den bisherigen Aufzeichnungen des in Rede stehenden Falls ein Resumé versuchen.

Da wir uns bisher weder aus den Moulages im Hospital St. Louis, noch aus der Beschreibung der einzelnen Autoren keine rechte Vorstellung von dieser Erkrankung machen konnten, sind wir derzeit, wo uns Gelegenheit geboten war, den Verlauf des Processes und die anatomischen Veränderungen kennen zu lernen, schon in der Lage ein klares Urtheil uns bilden zu können.

Wir glaubten anfangs ein Erythema toxicum, später nach erfolgter Eruption derber Knötchen einen Lichen ruber acuminatus vor uns zu haben, und erst zuletzt, nachdem der Verlauf der Knötchen ganz abweichend von denen des Lichen acuminatus sich erwies combinirt mit einer ganzen Reihe anderer Erscheinungen, rückten wir der Diagnose Pityriasis rubra pilaris näher. Welches sind nunmehr die Merkmale, welche zu Gunsten der letzteren sprechen?

1. Die Grösse und der ephemere Verlau f der Knötchen, welche hier punktförmig sind, mit dünnen Lamellen bedeckt, sich alsbald nach ihrem Entstehen wieder abflachen mit Hinterlassung einer weichen, elastischen, nicht infiltrirten, blassroth gefärbten schuppenden Hautpartie. Die Efflorescenzen bieten demnach ganz entgegengesetzte Merkmale von jenen Knötchen dar, welche dem Lichen ruber angehören, stecknadelkopfgross, braunroth gefärbt, wachsartig glänzend, mit centraler Delle versehen, persistent und mit wenig Schuppen bedeckt sind, und welche wenn sie geschwunden, derbe, braunrothe, infiltrirte, chagrinlederähnliche, mit tiefen Furchen durchzogene Stellen zurücklassen und bei universeller Verbreitung Nutritionsstörungen, hochgradige Abmagerung unausbleiblich im Gefolge haben. 
Weiters kommt auch bei Lichen ruber die Affection der Nägel in Betracht, welche verdickt, uneben, gelbbraun entfärbt sind und an ihrem freien Rande sowohl, als auch auf ihrer Oberfläche brüchig werden, gleichwie hier Kopf-, Scham- und Achselhaare verschont bleiben.

Vergleicht man die Knötchen der Pityriasis pilaire, sind dieselben am Stamme fast nur punlstförmig, etwas grösser an den Vorderarmen und am grössten an den ersten Phalangen der Hand, woselbst sie entsprechend dem daselbst vorkommenden Haarkreise mehr als Hirsekorngrösse erreichen; am Stamme sind sie weiters mehr glatt, an den Vorderarmen mehr reibeisenartig. Auch ihr Vtrlauf ist ein verschiedener. Am raschesten flachen sie sich am Stamme $a b$, am persistentesten sind sie an den Extremitäten.

An der ersten Phalanx fällt aus der Mitte der Efflorescenz ein Schüppchen heraus, wobei eine kraterförmige Vertiefung zurückbleibt. Hier verbreiten sich die Knötchen mehr flächenförmig, stehen in Gruppen von 10 bis 20 an einander. Im Allgemeinen aber setzen die bei dieser Erkrankung vorkommenden Knötchen wenig Widerstand den erweichenden Salben oder dem kratzenden Nagel entgegen und werden durch dieselben leicht beseitigt.

Die Farbe der Knötchen war anfangs dunkelroth hämorrhagisch, gleichwie ganze Hautstrecken theils blassroth, theils ron Petechien durchsetzt.

Die Veränderungen an den Fusssohlen waren in verschiedenen Stadien verschieden: Im Beginne des Processes waren die rkrankten Stellen geröthet, mit zahlreichen hämorrhagischen Punkten durchsetzt, im weiteren Verlaufe war auch die Hohlhand mit dichtgedrängten derben Epidermismassen bedeckt, eine wahre Hyperkeratose, welche entsprechend den Interphalangealgelenken von Rhagaden durchzogen war.

Die Nägel waren durch neugebildete Nagelsubstanz, welche vom Nagelbette aus sich entwickelt, secundär afficirt, in die Höhe gehoben, seitlich comprimirt.

Subjectiv hatte der Kranke nicht zu leiden, kein Jucken, Appetit und Schlaf waren nicht gestört, die Ernährung hatte 
nicht abgenommen, Harn war frei von Eiweiss, nur leichte Spannung an den Fusssohlen und Handtellern, glexchwie etwas Frösteln bisweilen belästigen ihn in geringem Grade. Die Lymphdrüsen in der Achselhöhle waren etwas schmerzhaft geschwellt.

Auf den Verlauf der Krankheit hatte der Arsenik keinen wesentlichen Einfluss, dagegen der Gebrauch von Salben, von Salicylseifenpflaster, namentlich das Tragen ron vulcanisirter Kautschukleinwand, Einwicklung in nasse Tücher und lauwarme Bäder brachten bald eine Abflachung der Knötchen und Herabminderung der Spannung hervor, und schliesslich wurde die Haut normal gefärbt, weich und elastisch, die Hautfalten nicht infiltrirt, demnach verlief der ganze Process ganz abweichend von dem der Lichen ruber.

Aber auch der mikroskopische Befund ist ganz und gar different von dem beider Lichenarten.

Schon Karmanski, (s. Besnier, Annales de Dermatologie et Syphilographie 1889), Jacquet haben Hautstücke und C. Boeck die Epidermis untersucht und konnte letzterer vorwiegend Hypertrophie der letzteren constatiren, die sich in Lamellen ablöste, wäbrend Karmanski und Jacquet an den tieferen Theilen der Cutis keine in der oberen Lage leichte Rundzellenwucherung nachweisen konnten, wobei die Haarbälge mit verhornten Zellen erfüllt waren.

Ich habe dem in Rede stehenden Kranken kleine Hautstückchen exstirpirt und an mikroskopischen Durchschnitten, welche mein Assistent Dr. Cehak angefertigt, konnte ich die Befunde der französischen Autoren theils bestätigen, theils erweitern. Die rerhornte Schicht der Epidermis, in grossen Lamellen abgelöst, füllt vollständig die Mündungen der Schweissdrüsen aus, reicht auch in die Tiefe der Haarbälge, dieselben stellenweise erweiternd, bildet neben dem von seiner Papille abgelösten Haare und den Zellen der äusseren Wurzelscheide den vorwiegenden Inhalt des Haarbalges. Eine besondere Beachtung verdient das Verhalten der Lamellen. Die verhornten Schichten liegen säulenförmig über einander, und sind wie bei allen Hypertrophien der Epidermis unmittelbar der Stachelzellenschicht aufgelagert, während die granulirte und die sogenannte 
Oel'sche Schicht vollständig zu fehlen scheint. Dagegen sind die Cylinderzellen in normalem Verhältniss auf den vergrösserten Papillen aufgelagert.

Die Haarbalgdrüsen sind normal, über deren Inhaltszellen lässt sich, da dieselben durch die Präparationsmethode verändert sind, nichts weiter angeben, jedoch sind die Wandungen der Drüsen leicht infiltrirt, die Schweissdrüsen sind in ihren Mündungen erweitert, bieten aber sonst nichts wesentlich Abnormes dar.

In dem Cutisgewebe finden sich an den Blutgefässen, welche leicht hyperämisch sind, einzelne Rundzellen; zerstreut im Cutisgewebe zeigen sich einzelne Proliferationen.

Vergleicht man diesen Befund mit jenem rom Lichen planus und Lichen acuminatus ist der Unterschied ein beträchtlicher. Bei der Pityriasis pilaris ist vorwiegend das Epidermisstratum ergriffen, während bei den Lichenarten das Cutisgewebe, allerdings vorwiegend in seinen oberen Lagen in dem Stratum vasculosum nur leichte Entzündungserscheinungen zeigt.

Hypertrophien des Cutisgewebes, das Auftreten von pigmentirten Bindegewebskörperchen zumal an der Adventitia der Gefässe und in den Schweissdrüsen fehlten hier vollständig. Somit haben wir es bei der Pityriasis pilaire mit einem acuten erythematösen, mehr in den oberen Lagen der Cutis auftretenden Processe zu thun, welcher theils mit Hyperkeratose, theils mit Exfoliation der Epidermis endigt und vorwiegend die Hautfollikel zumal die Haarbälge trifft. Auf ein Moment muss hier besonders hingewiesen werden. Bekanntlich tritt bei dem Lichen ruber eine beträchtliche Zunahme der Zellen der äusseren Wurzelscheide ein, welche sich an der Basis des Haarbalges ansammeln und demselben häufig den bekannten acinösen Bau verleihen. Bei der in Rede stehenden Erkrankung dagegen ist es mehr die innere Wurzelscheide, welche an Masse zunimmt, den Haarbalg ausfiullt und das Haar von seiner Papille abluebt.

Die eben angeführten Merkmale der Knötchenbildung, deren ephemerer Bestand, die glatte Oberfläche und die normale Farbe der Haut nach Schwund der Efforescenzen, die geringen subjectiven Erscheinungen, das Fehlen jeder ernsten 
Ernährungsform und da jede ernstere Complication ausgeschlossen werden kann, nicht minder der histologische Befund sprechen in solch überzeugender Weise dafür, dass der Lichen ruber hier mit Bestimmtheit ausgeschlossen werden kann.

9. Mai 1891. Nachdem der Kranke bis jetzt 72 Einspritzungen mit Acid. arsenicosum gebraucht hatte, wobei häufig Intoxicationserscheinungen anfgetreten waren, nachdem weiters das Tragen eines Kautschukanzuges gleichwie der Gebrauch von Damptbädern, Einwickelungen und Einölungen alternirend in Anwendung kamen, zeigen sich derzeit folgende Veränderungen :

Die ganze Hautoberfläche ist glatt, die Bildung von Schuppen ist sistirt, die Röthung ist vorwiegend an den Extremitäten vorhanden, während am Stamme, Rücken und Brust einzelne Hautpartien ganz weiss geblieben sind. Die Haut ist weich, elastisch, an den gerötheten Stellen ist die abgehobene Falte noch etwas verdickt. Zahlreiche umschiebene weisse Flecke von Linsenund Silbergroschengrösse, die noch vor 4 Wochen vorhanden waren, sind derzeit geschwunden, neue sind wieder entstanden. Die Nägel sind allenthalben an ihrer Oberfläche glatt, stellenweise scheint das dunkel geröthete Corium durch die hypertrophische Nagelsubstanz geschwunden, so dass der freie Rand des Nagels wieder normal geworden. Das Körpergewicht hat um $4 \frac{1}{2} \mathrm{~kg}$ zugenommen und ist das Befinden des Kranken im Ganzen ein gutes.

Es ergiht sich nach diesem Befunde, dass dieser Fall in allen seinen Symptomen übereinstimmt mit der von der französischen Schule beschriebenen Pityriasis pilaire.

Die lange Dauer der Krankheit, die Bildung von punktförmigen Knötchen, welche aus Epıdermishügelchen bestehen, die Gruppirung der Knötchen an der dritten Phalanx eutsprechend den einzelnen daselbst vorkommenden Haaren, die anatomischen Veränderungen, die Glätte der Haut, gleichwie die geringen Störungen der Ernährung sprechen zu Gunsten der Piteriasis rubra pilaire. Ein endgiltiges Urtheil dagegen wird erst dann möglich sein, wenn sich mehrere Fälle dieser Art der Beobachtung darbieten, aber schon jetzt ist es sicher, dass sowohl der Lichen ruber acuminatus als auch der Lichen planus, 
obzwar diese in ihrer Configuration mit Pityriasis pilaire viel Aehnlichkeit haben, von der in Rede stehenden Erkrankung verschieden sind.

Es gibt somit einen Lichen ruber acuminatus, einen $\mathrm{Li}$ chen planus und eine Pityriasis rubra pilaris, die beiden ersteren als zusammengehörige, die letztere als von denselben unabhängige Erkrankung.

\section{Erklärung der Abbildungen auf Taf. I-III.}

Taf. I. Der Process in den ersten Stadien seiner Entwicklung.

Taf. II. Der Process auf dem Höhepunkte seiner Entwicklung mit den charakteristischen miliären Knötchen der Pityriasis rub. pil.

Taf. III. Senkrechter Schnitt durch ein diffus geröthetes Hautstück von Pityriasis rubra pilaris, nachdem die dichten Lagen verhornter Zellen vorher entfernt wurden.

a Verhornte Epidermis.

$b b b$ Rete Malpighii, c Wollhaar, $d$ hyperämische Gefässe.

e Zerstreut liegende Rundzellen. 


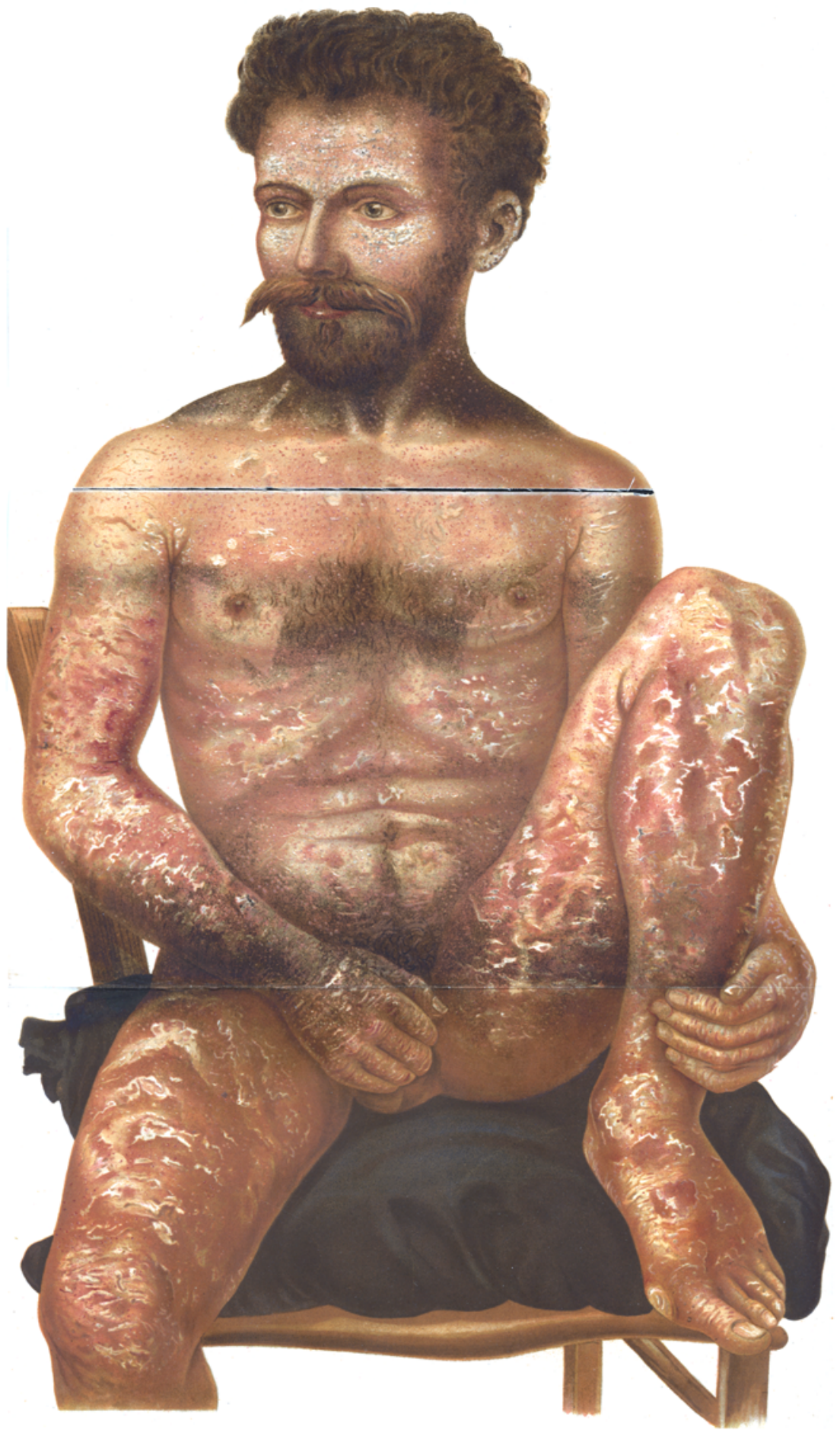




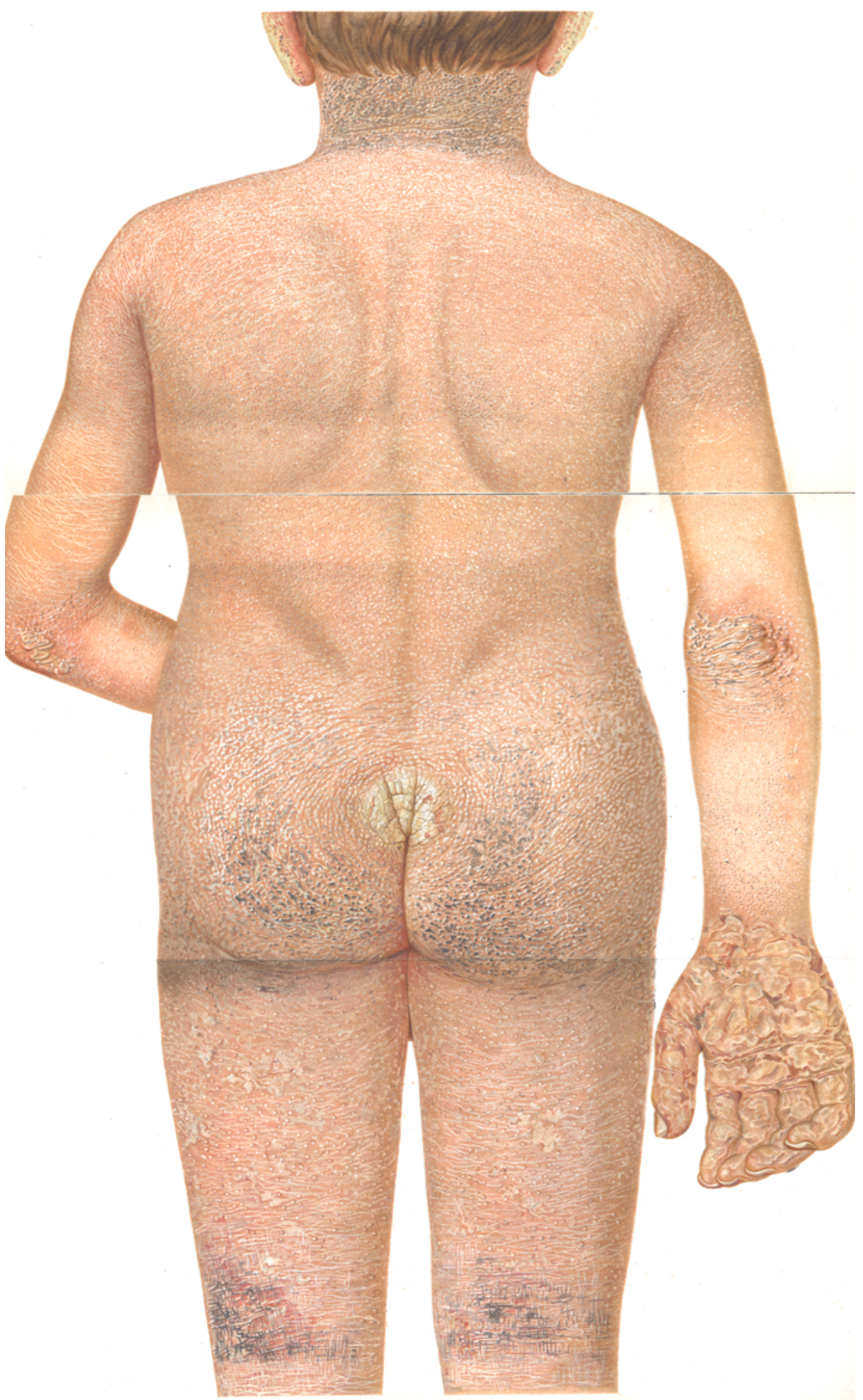




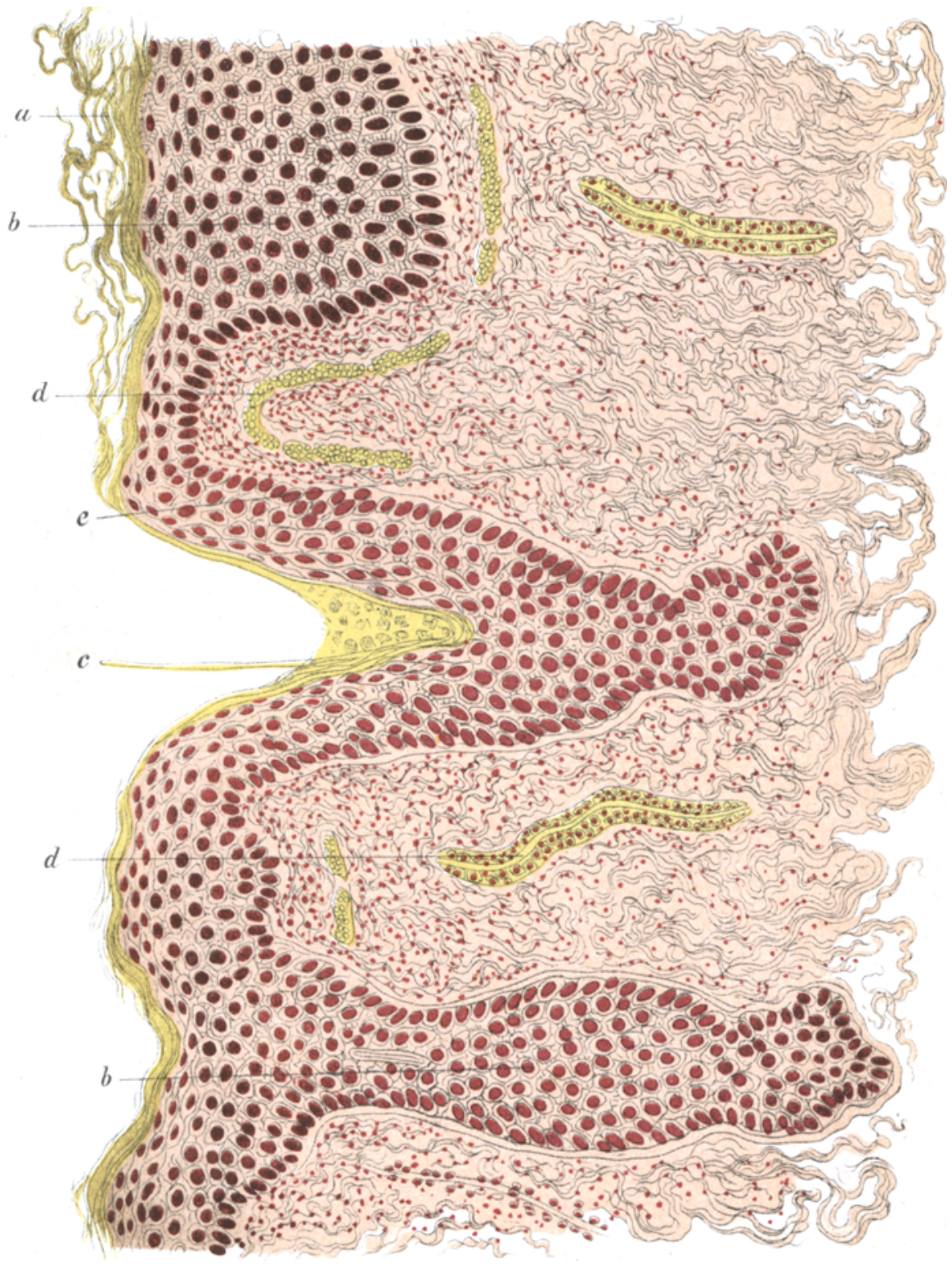

